Check for updates

Cite this: RSC Adv., 2017, 7, 19611

Received 28th February 2017

Accepted 27th March 2017

DOI: $10.1039 / c 7 r a 02464 a$

rsc.li/rsc-advances

\section{Effects of dietary fiber on the antioxidant capacity, immune status, and antioxidant-relative signaling molecular gene expression in rat organs}

\author{
Tingting Fang, $\uparrow^{\mathrm{ab}}$ Xianjian Wu, $\dagger^{\mathrm{ab}}$ Wei Cao, $\uparrow^{\mathrm{ab}}$ Gang Jia, ${ }^{\mathrm{ab}}$ Hua Zhao, ${ }^{\mathrm{ab}}$ \\ Xiaoling Chen, ${ }^{\text {ab }}$ Caimei Wu, ${ }^{\text {ab }}$ Jiayong Tang, ${ }^{\text {ab }}$ Jing Wang $^{\mathrm{c}}$ and Guangmang Liu (D) *ab
}

This study was conducted to evaluate the effects of different fibers, such as dietary pea, sweet potato, and wheat bran fibers, on the antioxidant capacity, immune status, and antioxidant-related signaling molecules of rat organs. In a 30 day experiment, a total of 44 rats were randomly divided into four dietary groups. These rats were fed either with a control diet or three basal diets supplemented with $15 \%$ pea fiber, $15 \%$ sweet potato fiber, and 15\% wheat bran fiber. Results are as follows: (1) different fibers, such as dietary pea, sweet potato, and wheat bran fibers, can effectively promote antioxidant defense by decreasing free radicals and increasing non-enzymatic and enzymatic antioxidant capacities in relevant organs $(P<0.05)$. (2) Pea, sweet potato, and wheat bran fiber significantly increase the mRNA levels of enzymatic antioxidant substances in relevant organs. Moreover, supplementation of these fibers remarkably increased the NF-E2-related nuclear factor 2, Kelch-like ECH-associated protein 1, and mammalian target of rapamycin in the spleen $(P<0.05)$. (3) These fibers also significantly upregulated the antiinflammatory mRNA level but downregulated the pro-inflammatory cytokine mRNA level in the jejunum, liver, and spleen. The mRNA level of transforming growth factor beta 1 also increased in rats with wheat bran fiber in different tissues $(P<0.05)$. In summary, pea fiber, sweet potato fiber, and wheat bran fiber supplements can improve the antioxidant capacity of plasma and relevant organs and regulate antioxidant-related signaling molecular expression in the jejunum, liver, and spleen of rats. Various fibers exerted different effects on immunity function in the jejunum, liver, and spleen by mediating the gene expression levels of anti-inflammatory and pro-inflammatory cytokines, TGF- $\beta$, and caspase 3 .

\section{Introduction}

In recent years, a rapid increase in lifestyle diseases has resulted in an increasing awareness about the health benefits of dietary fiber (DF). DF contains natural food sources, processed grains, and commercial supplements. ${ }^{1}$ Among DFs, pea fiber (PF), sweet potato fiber (SF), and wheat bran fiber (WF) are increasingly used for human food and animal diets. Many studies have revealed that these fibers show potential as excellent novel sources of nutritious food because of their techno-functional properties and significance in promoting health.

Fiber-rich diets might help prevent many nutritional diseases and promote healthy activities, such as reduction of risk of gastrointestinal-related problem, oxidative stress, and

\footnotetext{
${ }^{a}$ Institute of Animal Nutrition, Sichuan Agricultural University, Chengdu 611130, Sichuan, China. E-mail: liugm@sicau.edu.cn; Tel: +86-28-86290976

${ }^{b}$ Key Laboratory for Animal Disease-Resistance Nutrition of China Ministry of Education, Chengdu 611130, Sichuan, China

${ }^{\prime}$ Maize Research Institute, Sichuan Agricultural University, Chengdu 611130, Sichuan, China

$\dagger$ Contributed equally.
}

immune disorder. ${ }^{2}$ Dietary supplementation with PF, SF, and WF can promote positive health effects by changing certain hemal systemic metabolic processes, regulating intestinal microbiota, and affecting gut barrier function..$^{3-5}$ The protective function of plasma and digestive organs is involved in the antioxidant status. However, few studies have been carried out to examine the antioxidative effects of fiber source intake with different fiber components (PF, SF, and WF) on plasma and other relevant organs (liver, intestine, and spleen). Pea, sweet potato, and wheat bran exhibit increased free radicalscavenging activities and prevent low-density lipoprotein cholesterol oxidation..$^{6-9}$ The effects of PF, SF, and WF on antioxidant defense systems, including enzymatic antioxidant and non-enzymatic antioxidant activities of plasma and other relevant organs in animal, are not well appreciated. Moreover, the enzymatic antioxidant defense systems are activated through a multitude of biochemical mechanisms, including increased antioxidant enzyme-related gene expression and stimulation of the nuclear erythroid 2-related factor 2 (Nrf2) pathway. ${ }^{10,11}$ Nrf2 is sequestered in the cytoplasm by the actin-binding protein Kelch-like ECH-associated protein 1 (Keap1). ${ }^{10}$ The Nrf2-Keap1 system is a key molecular target of cellular enzymatic 
antioxidant inducers. ${ }^{11}$ Hwang et al. ${ }^{12}$ reported that anthocyanins from purple sweet potato can increase the expression of antioxidant enzyme and Nrf2. Nevertheless, the antioxidant enzyme-related gene expression and Nrf2-Keap1 signaling pathways involved in the induction of $\mathrm{PF}, \mathrm{SF}$, and WF of antioxidant enzymes against oxidative damage remain not completely understood. Nuclear Nrf2 accumulation may be regulated by the mammalian target of rapamycin (mTOR). ${ }^{13}$ Nevertheless, the mechanism by which PF, SF, and WF elicit antioxidant effect in association with mTOR in relevant organs is generally unknown. Additionally, the role of different fiber sources in preventing the deleterious consequences of oxidant status remains unclear. Hence, further studies are also needed.

DF can modulate various inflammatory processes and innate immune systems. DF can regulate the immune system by changing T-helper (Th) cell polarization and influencing Th 1/2determining cytokines. Th1-type cells primarily secrete high levels of interferon- $\gamma$, interleukin (IL)-2, IL-6, and tumor necrosis factor (TNF)- $\beta$, which promote cell-mediated immune responses; Th2-type cells produce a different set of antiinflammatory cytokines and secrete IL-4, IL-10, and IL-13, which promote humoral immunity. ${ }^{14}$ In vivo results showed that plasma IL-1 $\beta$ content is numerically, but not significantly reduced; however, IL-8 is reduced in overweight/obese adults with the intake of yellow PF. ${ }^{15}$ Moreover, pigs supplemented with WF and PF diets exhibit low IL-1 $\alpha$ mRNA levels in the colon compared with those on maize fiber and soya bean fiber diets. ${ }^{5}$ Whether supplementation with PF, SF, and WF can actually affect the inflammatory cytokine gene expression in jejunum, liver, and spleen remains unclear. Notably, recent studies suggested that wheat bran treatment can upregulate the nuclear factor $\kappa \mathrm{B}$ in stomach and jejunum, as well as TNF- $\alpha$, transforming growth factor beta (TGF- $\beta$ ), and caspase 3 in jejunum. ${ }^{\mathbf{1 6}}$ Similarly, both TGF- $\beta$ and caspase 3 play a central role in the executory phase of cell apoptosis, cellular homeostasis, and other cellular functions. ${ }^{17}$ Nonetheless, the effect of different dietary fiber sources on these gene expression levels in jejunum, liver, and spleen is yet to be determined. The immunological effect among PF, SF, and WF is also not yet established.

The present study is part of a large research project that determines the various effects of PF and WF supplementation in rat metabolism. ${ }^{3}$ This study primarily aimed to examine the effect of PF, SF, and WF on radical scavenging, enzymatic antioxidant, and non-enzymatic antioxidant activities of plasma, jejunum, liver, and spleen. Furthermore, we investigated the antioxidant effects of those fibers on the gene expression levels of antioxidant-related signaling molecules and inflammatory cytokines of the jejunum, liver, and spleen in rats. The present results will elucidate the immunological functions, antioxidant properties, and underlying cellular mechanisms of oxidative response to $\mathrm{PF}, \mathrm{SF}$, and $\mathrm{WF}$ supplementation in the jejunum, liver, and spleen of rats. An understanding of the influences of PF, SF, and WF will provide useful insights on utilization of $\mathrm{PF}, \mathrm{SF}$, and WF for healthy living and in addressing various nutritional and dietrelated disorders.

\section{Materials and methods}

\section{Materials}

PF was provided by Shandong Jianyuan Foods Co., Ltd (Shandong, China), SF was from Shanxi Sciphar Hi-tech Industry Co., Ltd. (Shanxi, China), and WF was from Sichuan Foods Co., Ltd (Sichuan, China). Female Sprague-Dawley rats with a body weight (BW) of $170 \mathrm{~g}$ to $201 \mathrm{~g}$ were purchased from the Dossy Experimental Animals Co., Ltd (Chengdu, China). Basic diets were formulated referring to our previous studies, which can conform the nutrient recommendations of the AIN 93 for laboratory rodents. ${ }^{18}$ The compositions of basic diet are shown in Table 1. All antioxidative reagent kits used in the present study were purchased from Nanjing Jiancheng Bioengineering Institute (Nanjing, China).

\section{Animal experiments and sample collection}

Animals were maintained according to the National Research Council's Guide for the Care and Use of Laboratory Animals and approved by the Care and Use Ethical Committee of Sichuan Agricultural University of China. After one week of acclimatization, a total of 44 eight-week-old female Sprague-Dawley rats were randomly divided into four dietary groups (i.e., $\mathrm{C}, \mathrm{PE}, \mathrm{SF}$, and WF) with 11 replicates per group. The experimental diets included a control diet without a supplemental fiber source and three basal diets supplemented with 15\% PF, 15\% SF, and 15\% WF. The rats were housed in individual metabolic cages in a nursery room. Room temperature and humidity were $25{ }^{\circ} \mathrm{C}$ and $60 \%$, respectively. A $12 \mathrm{~h}$ light and $12 \mathrm{~h}$ dark cycle was maintained during the 30 day experimental period. All rats were provided ad libitum access to water and fed with their respective diets.

Rats were anesthetized with ether after overnight fasting at the end of the 30 day treatment period. Blood samples from the orbital venus plexus were collected (09:00 a.m.) into Eppendorf tubes containing sodium heparin. The tubes were centrifuged at $3500 \times g$ for $10 \mathrm{~min}$ at $4{ }^{\circ} \mathrm{C}$. Subsequently, the plasma samples were harvested and stored at $-80{ }^{\circ} \mathrm{C}$ until further testing. After blood sampling, all rats were then sacrificed to collect the jejunum, liver, and spleen samples. The jejunum, liver, and spleen samples were snap frozen in liquid nitrogen and then stored at $-80^{\circ} \mathrm{C}$ for analyses of antioxidant status and immune cytokines.

\section{Determination of antioxidant index in the plasma, jejunum, liver, and spleen}

To evaluate the antioxidant status in plasma, jejunum, liver, and spleen, we determined the glutathione (GSH) contents and the antisuperoxide anion (ASA), antihydroxyl radical (AHR), total antioxidant capacity (T-AOC), total superoxide dismutase (T-SOD), and catalase (CAT) activities. Frozen jejunum, liver, and spleen were weighed, thawed, and homogenized at 10 times the volume $(\mathrm{w} / \mathrm{v})$ of ice-cold physiological saline. The mixture was centrifuged at $6000 \times g$ at $4{ }^{\circ} \mathrm{C}$ for $10 \mathrm{~min}$. Subsequently, the supernatants were collected and stored at $-20{ }^{\circ} \mathrm{C}$ for parameter analysis. 
Table 1 Composition and nutrient level of the experimental diets ${ }^{a}$

\begin{tabular}{|c|c|c|c|c|}
\hline \multirow[b]{2}{*}{ Items } & \multicolumn{4}{|c|}{ Fiber sources } \\
\hline & Control & Pea fiber & $\begin{array}{l}\text { Wheat } \\
\text { bran fiber }\end{array}$ & $\begin{array}{l}\text { Sweet } \\
\text { potato fil }\end{array}$ \\
\hline \multicolumn{5}{|l|}{ Ingredients (\%) } \\
\hline Corn starch & 44.74 & 31.92 & 31.92 & 31.92 \\
\hline Casein & 20.00 & 20.00 & 20.00 & 20.00 \\
\hline Corn amylodextrin & 13.20 & 13.20 & 13.20 & 13.20 \\
\hline Sucrose & 10.00 & 10.00 & 10.00 & 10.00 \\
\hline Soybean oil & 7.00 & 4.82 & 4.82 & 4.82 \\
\hline Pea fiber & 0.00 & 15.00 & 0.00 & 0.00 \\
\hline Sweet potato fiber & 0.00 & 0.00 & 0.00 & 15.00 \\
\hline Wheat bran fiber & 0.00 & 0.00 & 15.00 & 0.00 \\
\hline L-Cysteine & 0.30 & 0.30 & 0.30 & 0.30 \\
\hline Mineral premix & 1.00 & 1.00 & 1.00 & 1.00 \\
\hline Vitamin premix & 3.50 & 3.50 & 3.50 & 3.50 \\
\hline Choline chloride (50\%) & 0.25 & 0.25 & 0.25 & 0.25 \\
\hline Antioxidant & 0.01 & 0.01 & 0.01 & 0.01 \\
\hline Total & 100.00 & 100.00 & 100.00 & 100.00 \\
\hline \multicolumn{5}{|l|}{ Nutrient content } \\
\hline Gross energy $\left(\mathrm{kcal} \mathrm{kg}^{-1}\right)$ & 3766.00 & 3766.00 & 3766.00 & 3766.00 \\
\hline Crude protein $(\%)$ & 15.20 & 15.20 & 15.20 & 15.20 \\
\hline Crude fiber (\%) & 3.35 & 2.88 & 1.38 & 5.59 \\
\hline Neutral detergent fiber (\%) & 22.82 & 27.40 & 13.81 & 17.54 \\
\hline Acid detergent fiber (\%) & 3.43 & 3.39 & 2.83 & 3.34 \\
\hline Cellulose $(\%)$ & 0.55 & 1.54 & 0.88 & 1.52 \\
\hline Hemicellulose (\%) & 0.05 & 14.20 & 11.40 & 14.20 \\
\hline Lignin (\%) & 0.81 & 1.43 & 1.39 & 0.60 \\
\hline Soluble fiber (\%) & 0.04 & 0.02 & 0.05 & 15.00 \\
\hline Insoluble fiber (\%) & 0.03 & 0.08 & 0.04 & 0.30 \\
\hline Total fiber (\%) & 0.07 & 0.10 & 0.09 & 15.30 \\
\hline
\end{tabular}

${ }^{a}$ Vitamin premix provided per kg diet: vitamin A, 0.80 g; vitamin D3, $0.20 \mathrm{~g}$; vitamin E, $15.00 \mathrm{~g}$; vitamin $\mathrm{K}, 0.17 \mathrm{~g}$; vitamin $\mathrm{B} 12,0.25 \mathrm{~g}$; riboflavin, $0.75 \mathrm{~g}$; niacin, $3.06 \mathrm{~g}$; pantothenic acid, $1.67 \mathrm{~g}$; folic acid, $0.21 \mathrm{~g}$; thiamin, $0.62 \mathrm{~g}$; pyridoxine, $0.74 \mathrm{~g}$; biotin, $1.00 \mathrm{~g}$. Mineral premix provided per $\mathrm{kg}$ diet: $6.88 \mathrm{~g} \mathrm{Fe}\left(\right.$ as $\mathrm{FeSO}_{4} \cdot 7 \mathrm{H}_{2} \mathrm{O}$ ); $0.30 \mathrm{~g} \mathrm{Cu}$ (as $\left.\mathrm{CuCO}_{3}\right) ; 1.65 \mathrm{~g} \mathrm{Zn}\left(\right.$ as $\left.\mathrm{Zn}_{2}(\mathrm{OH})_{2} \mathrm{CO}_{3}\right) ; 0.83 \mathrm{~g} \mathrm{Mn}\left(\right.$ as $\left.\mathrm{MnSO}_{4} \cdot \mathrm{H}_{2} \mathrm{O}\right) ; 0.1 \mathrm{~g}$ $\mathrm{I}$ (as KI); $0.01 \mathrm{~g}$ Se (as $\mathrm{Na}_{2} \mathrm{SeO}_{3}$ ).

The analysis methods of plasma, jejunum, liver, and spleen oxidant status and the antioxidant enzyme and non-enzyme antioxidant activities were the same as our previous study. ${ }^{19}$ Briefly, the ASA and AHR capacities were determined by the method described by Jiang et al. ${ }^{20}$ superoxide radicals $\left(\mathrm{O}_{2}{ }^{-}\right)$ were generated by the reaction of xanthine and xanthine oxidase. The coloration degree can directly express the amount of superoxide anion in the reaction. Hydroxyl free radicals $\left(\mathrm{OH}^{-}\right)$were generated on the basis of the Fenton reaction $\left(\mathrm{Fe}^{2+}+\right.$ $\left.\mathrm{H}_{2} \mathrm{O}_{2} \rightarrow \mathrm{Fe}^{3+}+\mathrm{OH}^{-}+\mathrm{OH}\right)$, and the coloration degree was directly proportional to the quantity of hydroxyl radicals. The GSH content was measured according to the method of Vardi et $a l .{ }^{21}$ and the quantity of GSH produced was assayed in terms of the formation of 5-thio-2-nitrobenzoate and detected spectrophotometrically at $412 \mathrm{~nm}$. The T-AOC was measured using the colorimetric technique following the protocol of Wan et al. $;^{22}$ and detected absorbance value at $520 \mathrm{~nm}$ with colored and stable chelates when combined with phenanthroline. The T-SOD activity was assayed as described by Zhang et al.; ${ }^{23}$ and one unit of T-SOD was defined as the quantity of enzyme required to produce $50 \%$ inhibition of nitric ion production. The CAT activity was measured through decomposition of hydrogen peroxide according to a procedure described by Aebi et $a l . ;^{24}$ and one unit was defined as the amount of enzyme required to decompose $1 \mathrm{mmol} \mathrm{L}^{-1} \mathrm{H}_{2} \mathrm{O}_{2}$ within $1 \mathrm{~s}$ per milligram of tissue protein at $37^{\circ} \mathrm{C}$.

\section{Real-time quantitative PCR analysis of gene expression in the jejunum, liver, and spleen}

Total RNA was extracted from the jejunum, liver, and spleen using a TRIzol Reagent (Takara, Dalian, China) according to the manufacturer's protocol. The quality of total RNA was electrophoresed on $1.0 \%$ agarose gel electrophoresis (Beckman DU800; CA, USA). The quantity of all samples was checked by spectrophotometric analysis at 260 and $280 \mathrm{~nm}$, and the ratios of absorption (260/280 $\mathrm{nm})$ ranged between 1.8 and 2.0. Subsequently, total RNA was reverse transcribed into cDNA using the PrimeScript ${ }^{\mathrm{TM}}$ RT reagent (Takara, Dalian, China) following the manufacturer's instructions. The primers for $\beta$ actin, SOD1, CAT, $\mathrm{GP}_{X} 1$, glutathione reductase (GR), Nrf2, Keap1 , mTOR, IL- $1 \beta$, IL-6, IL-10, TNF- $\alpha$, TGF- $\beta 1$, and caspase 3 genes were designed with Primer Express Software (version 3.0; Applied Biosystems, Foster City, CA, USA) and synthesized by TaKaRa Biotechnology Company (Takara, Dalian, China). The primer sequences of the corresponding genes and the optimal annealing temperatures are summarized in Table 2. All realtime PCR reactions were performed with the SYBR® Green I PCR Reagent Kit and a real-time PCR instrument (ABI 7900HT, Applied Biosystems, USA). Amplification was performed at a final volume of $8 \mu \mathrm{L}$, which contained $4 \mu \mathrm{L}$ of SYBR Premix Ex Taq II with the ROX reference dye, $0.8 \mu \mathrm{L}$ of cDNA, $1.6 \mu \mathrm{L}$ of primer pairs, and $1.6 \mu \mathrm{L}$ of $\mathrm{ddH}_{2} \mathrm{O}$. The thermal cycling conditions for PCR initiated with a prerun step at $95{ }^{\circ} \mathrm{C}$ for $10 \mathrm{~s}, 42$ cycles of denaturation at $95{ }^{\circ} \mathrm{C}$ for $10 \mathrm{~s}$, annealing at $58{ }^{\circ} \mathrm{C}$ for $35 \mathrm{~s}$, and extension at $72{ }^{\circ} \mathrm{C}$ for $15 \mathrm{~s}$. The expression results were analyzed using the $2^{-\Delta \Delta C_{\mathrm{t}}}$ method, as described by Livak et al. ${ }^{25}$

\section{Statistical analysis}

All data were subjected to one-way ANOVA, which was followed by Duncan's multiple range test of SPSS 22.0 (SPSS Inc., Chicago, IL, USA). The results were expressed as mean \pm standard error. Significant differences among treatment groups were identified at the level of $P<0.05$.

\section{Results}

Antioxidant indicators in the plasma, jejunum, liver, and spleen

Table 3 presents the antioxidant indicators in the plasma. PF significantly decreased the AHR and T-SOD activities compared with the control and WF groups $(P<0.05)$. The AHR, T-AOC, TSOD, and CAT activities were lower, but the GSH content was higher in the SF group compared with those in the control group $(P<0.05)$. The T-SOD and CAT activities were the lowest in rats fed with SF diet than those fed with other diets $(P<0.05)$. Moreover, GSH content was enhanced, but T-AOC was 
Table 2 Primers sequences of target genes selected for analysis by real-time PCR

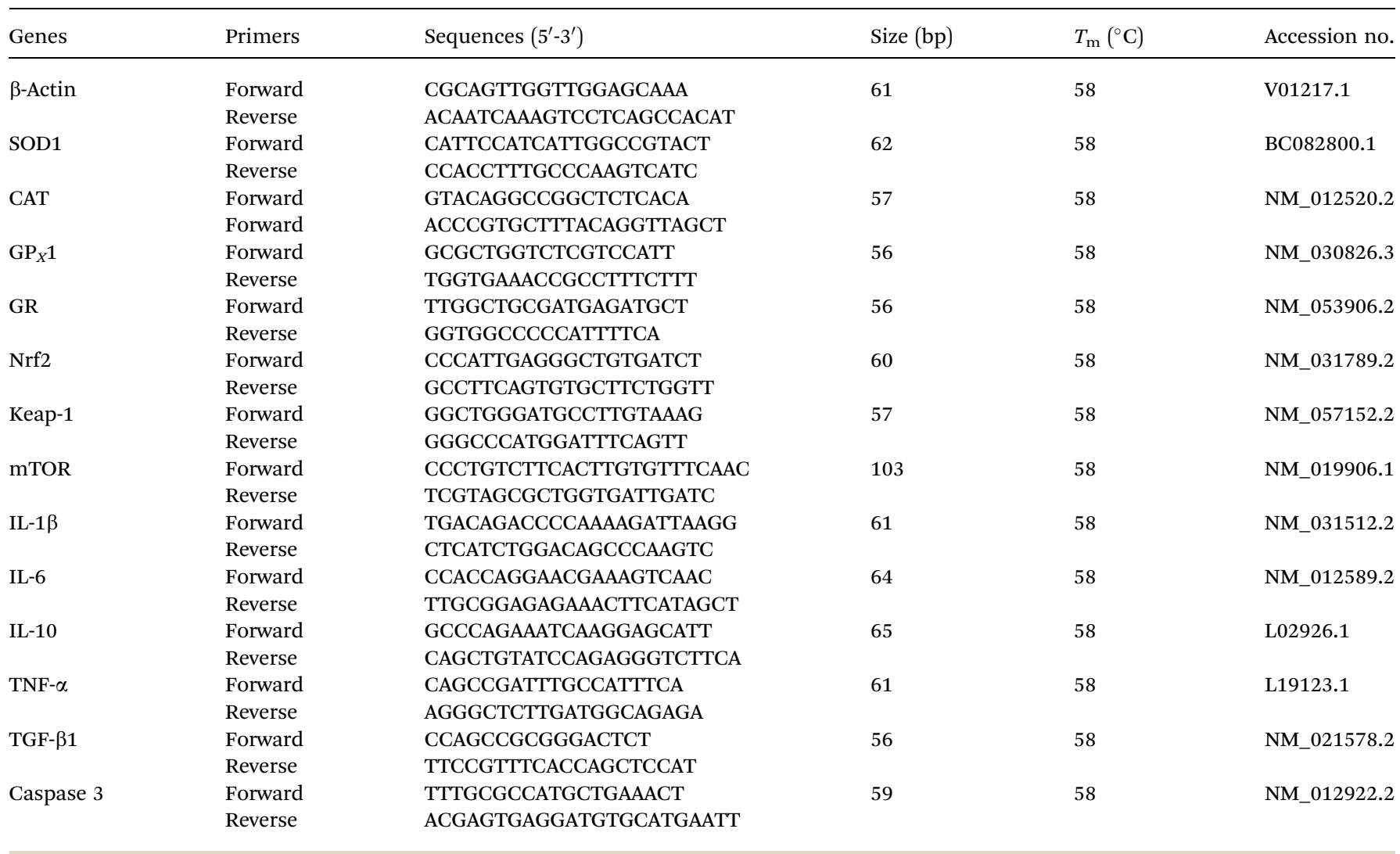

decreased by dietary WF supplementation relative to the control group $(P<0.05)$. Rats on WF diet exhibited the highest AHR, TSOD, and CAT activities compared with those fed with other diets $(P<0.05)$.

As shown in Table 4, the ASA, T-AOC, T-SOD, and CAT activities in jejunum were significantly increased by dietary $\mathrm{PF}$, $\mathrm{SF}$, and WF supplementations relative to the control group $(P<$ 0.05). A higher CAT activity and a lower GSH content were observed in rats in PF diet than in those in the SF diet $(P<0.05)$. Furthermore, rats supplemented with WF diet exhibited the highest ASA, AHR, T-SOD, and CAT activities than those in the $\mathrm{PF}$ and $\mathrm{SF}$ diets $(P<0.05)$.

Table 5 presents the antioxidant indicators in liver. PF significantly increased ASA, AHR, T-SOD, and CAT activities compared with the control group $(P<0.05)$. The lowest T-AOC activity was observed in the PF group compared with the SF and WF groups $(P<0.05)$. The ASA, AHR, and T-SOD activities were increased after SF supplementation. By contrast, the CAT activity in the liver decreased relative to the control group $(P<$ 0.05). Compared with the liver samples of the control group, the liver of WF-treated rats revealed a significant increase in the ASA, AHR, T-SOD, and CAT activities $(P<0.05)$. Higher ASA, AHR, and CAT activities were observed in rats fed with the WF diet compared with those in the SF diet $(P<0.05)$.

In the spleen (Table 6), the ASA activity and GSH content were increased, but T-SOD activity was decreased in rats fed with PF-supplemented diet compared with those under control diet $(P<0.05)$. Subsequently, the spleen of SF-treated rats

Table 3 Effects of pea fiber, sweet potato fiber, and wheat bran fiber on the antioxidant status of plasma in rats ${ }^{a, b}$

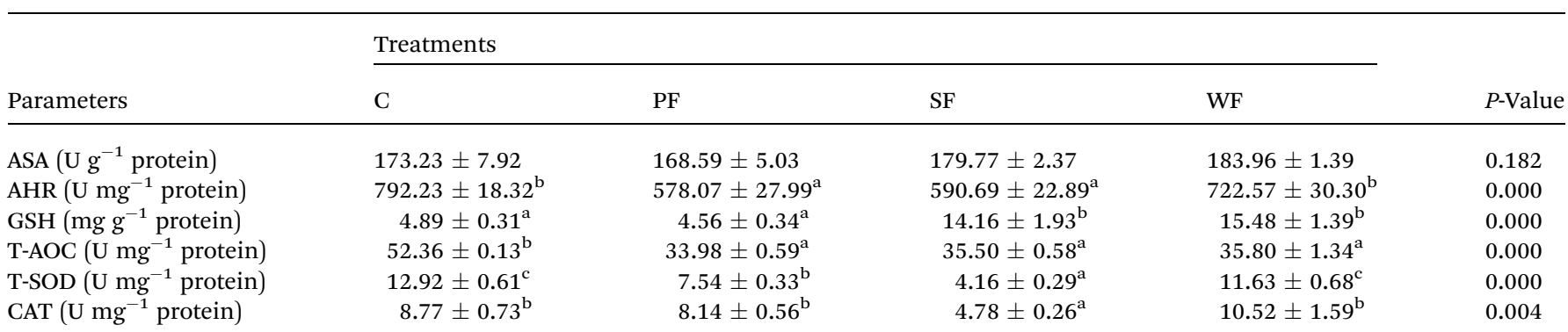

${ }^{a}$ Data are expressed as mean \pm SEM for eleven rats per treatment group. C, control; PF, pea fiber; SF, sweet potato fiber; WF, wheat bran fiber; ASA, antisuperoxide anion; AHR, antihydroxyl radical; GSH, glutathione; T-AOC, total antioxidant capacity; T-SOD, total superoxide dismutase; CAT, catalase. ${ }^{b-c}$ Mean values within a row with different superscript letters were significantly different $(P<0.05)$. 
Table 4 Effects of pea fiber, sweet potato fiber, and wheat bran fiber on the antioxidant status of jejunum in rats ${ }^{a, b}$



${ }^{a}$ Data are expressed as mean \pm SEM for eleven rats per treatment group. C, control; PF, pea fiber; SF, sweet potato fiber; WF, wheat bran fiber; ASA, antisuperoxide anion; AHR, antihydroxyl radical; GSH, glutathione; T-AOC, total antioxidant capacity; T-SOD, total superoxide dismutase; CAT, catalase. ${ }^{b \text { a-d }}$ Mean values within a row with different superscript letters were significantly different.

Table 5 Effects of pea fiber, sweet potato fiber, and wheat bran fiber on the antioxidant status of liver in rats ${ }^{a, b}$

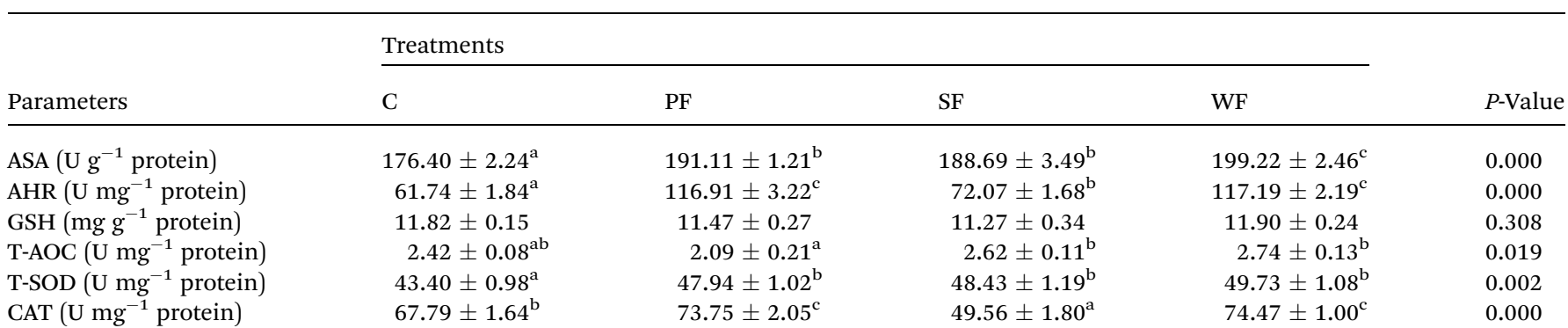

${ }^{a}$ Data are expressed as mean \pm SEM for eleven rats per treatment group. C, control; PF, pea fiber; SF, sweet potato fiber; WF, wheat bran fiber; ASA, antisuperoxide anion; AHR, antihydroxyl radical; GSH, glutathione; T-AOC, total antioxidant capacity; T-SOD, total superoxide dismutase; CAT, catalase. ${ }^{b \text { a-c }}$ Mean values within a row with different superscript letters were significantly different $(P<0.05)$.

revealed a notable increase in ASA activity, but a decrease in TAOC, T-SOD, and CAT activities relative to the control rats $(P<$ 0.05). In addition, WF supplementation obviously increased ASA, T-AOC, T-SOD, and CAT activities and GSH content relative to the control group $(P<0.05)$. Rats supplemented with $\mathrm{PF}$ and WF diets exhibited higher T-SOD and CAT activities and GSH content than those under SF diet $(P<0.05)$.

\section{mRNA levels of antioxidant-related parameters in the jejunum, liver, and spleen}

The mRNA levels of antioxidant-related parameters in the jejunum after PF, SF, and WF supplementations are listed in
Fig. 1. The expression of SOD1, CAT, $\mathrm{GP}_{X} 1$, and Keap-1 mRNA was significantly increased, but Nrf2 mRNA level was decreased in rats with $\mathrm{PF}$ supplementation compared with the control group $(P<0.05)$. Rats fed with SF diet showed higher SOD1, $\mathrm{GP}_{X} 1$, GR, and Keap-1 expression and lower Nrf2 mRNA level than those fed with the control diet $(P<0.05)$. Rats fed with SF diet exhibited the lowest CAT and Nrf2 mRNA levels compared with the other groups diets $(P<0.05)$. Moreover, WF significantly increased the mRNA levels of SOD1, CAT, GP 1 , GR, and Keap-1 compared with the control group $(P<0.05)$. WF group showed the highest Nrf2 mRNA level compared with the other groups $(P<0.05)$.

Table 6 Effects of pea fiber, sweet potato fiber, and wheat bran fiber on the antioxidant status of spleen in rats ${ }^{a, b}$

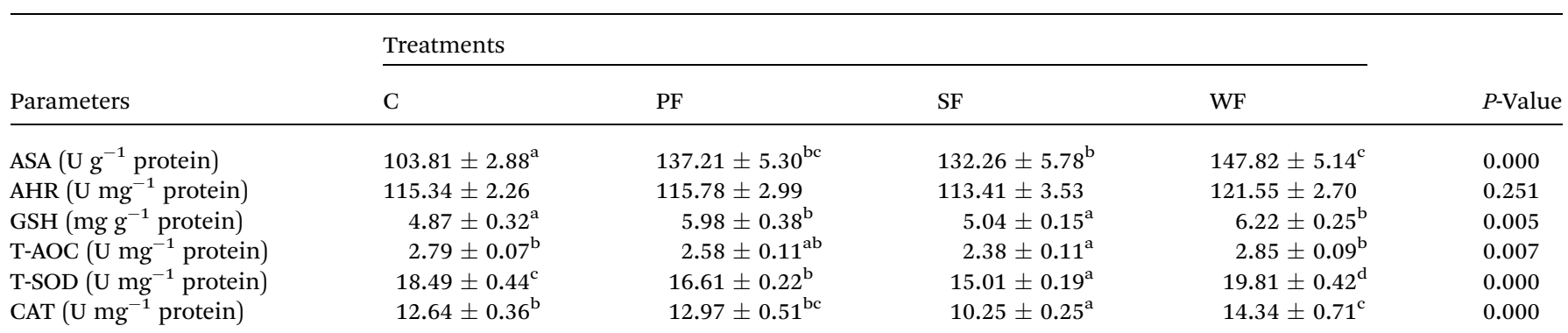

${ }^{a}$ Data are expressed as mean \pm SEM for eleven rats per treatment group. C, control; PF, pea fiber; SF, sweet potato fiber; WF, wheat bran fiber; ASA, antisuperoxide anion; AHR, antihydroxyl radical; GSH, glutathione; T-AOC, total antioxidant capacity; T-SOD, total superoxide dismutase; CAT, catalase. ${ }^{b-\mathrm{a}}$ Mean values within a row with different superscript letters were significantly different $(P<0.05)$. 


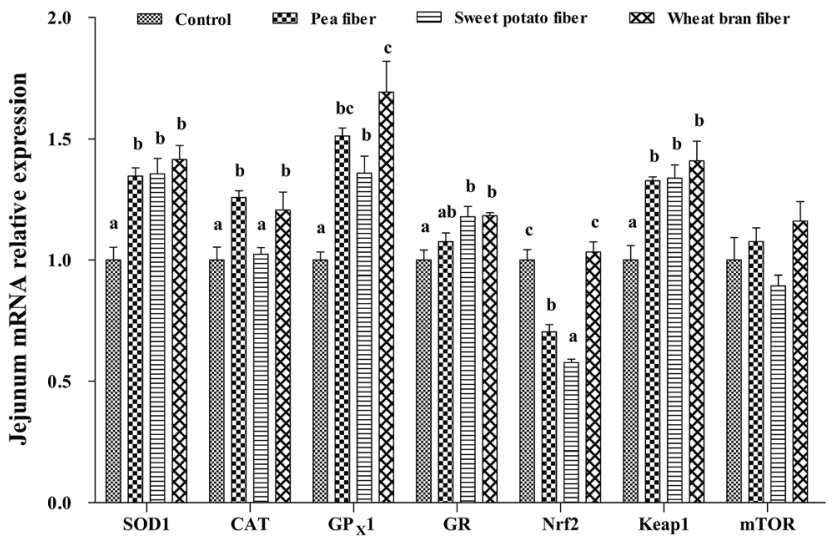

Fig. 1 Relative mRNA levels of SOD1, CAT, GP $\times 1$, GR, Nrf2, Keap1, and mTOR in the jejunum of rat fed diets with pea fiber, sweet potato fiber, and wheat bran fiber. SOD1, superoxide dismutase 1; CAT, catalase; $\mathrm{GP}_{x} 1$ glutathione peroxidase 1; GR, glutathione reductase; Nrf2, nuclear erythroid 2-related factor 2; Keap1, Kelch-like ECH-associated protein 1; and $\mathrm{mTOR}$, mammalian target of rapamycin. The values are the means $\pm \operatorname{SEM}(n=6){ }^{a, b, c}$ The different superscript letters above the bars show significant differences among the treatments $(P<0.05)$.

Compared with that in the control diet, the mTOR mRNA level in the liver was significantly increased, but the mRNA level of Keap-1 was decreased in response to PF-supplemented $\operatorname{diet}(P$ $<0.05$ ) (Fig. 2). Supplementing rats with SF resulted in a lower Keap-1 mRNA level compared with those of control rats $(P<$ 0.05). In addition, the CAT, $\mathrm{GP}_{X} 1, \mathrm{Nrf} 2$, and mTOR mRNA levels were higher in rats fed with WF-supplemented diet than those in the control diet $(P<0.05)$. Rats fed with WF diet showed higher CAT, $\mathrm{GP}_{X} 1, \mathrm{GR}$, Nrf2, and Keap-1 expression levels than those in the $\mathrm{SF}$ diet $(P<0.05)$.

The effects of dietary PF, SF, and WF on mRNA levels of antioxidant-related genes in the spleen are presented in Fig. 3.

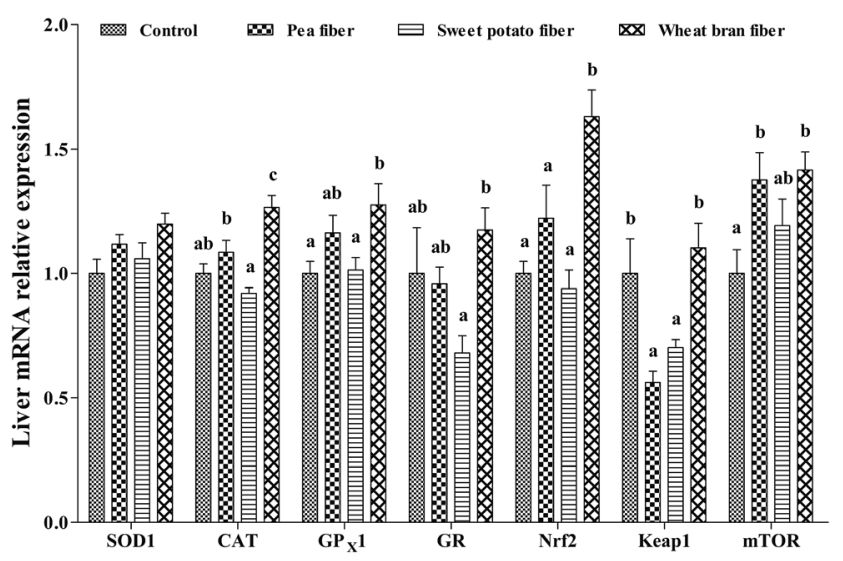

Fig. 2 Relative mRNA levels of SOD1, CAT, GP $x 1$, GR, Nrf2, Keap1, and mTOR in the liver of rat fed diets with pea fiber, sweet potato fiber, and wheat bran fiber. SOD1, superoxide dismutase 1; CAT, catalase; $\mathrm{GP}_{x} 1$ glutathione peroxidase 1; GR, glutathione reductase; Nrf2, nuclear erythroid 2-related factor 2; Keap1, Kelch-like ECH-associated protein 1; and mTOR, mammalian target of rapamycin. The values are the means $\pm \operatorname{SEM}(n=6) .{ }^{a, b, c}$ The different superscript letters above the bars show significant differences among the treatments $(P<0.05)$.

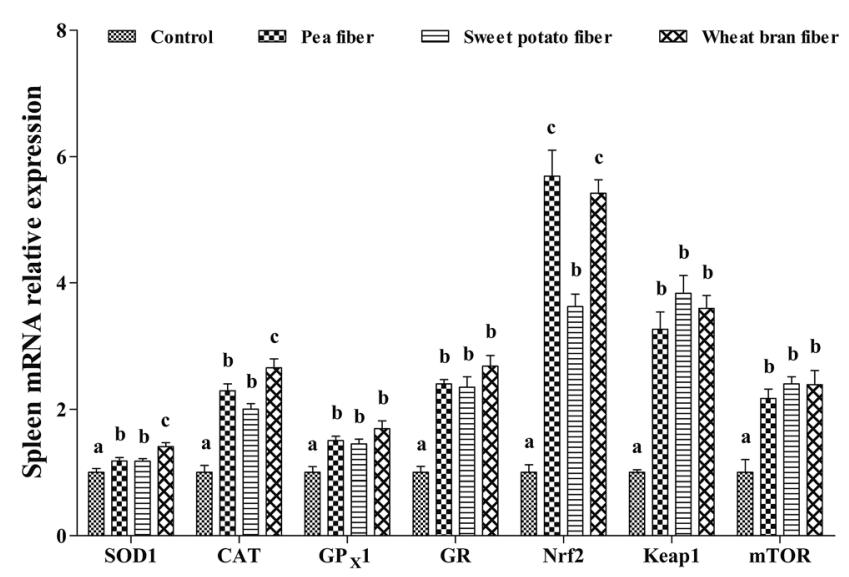

Fig. 3 Relative mRNA levels of SOD1, CAT, GP 1, GR, Nrf2, Keap1, and mTOR in the spleen of rat fed diets with pea fiber, sweet potato fiber, and wheat bran fiber. SOD1, superoxide dismutase 1; CAT, catalase; $\mathrm{GP}_{x} 1$ glutathione peroxidase 1; GR, glutathione reductase; Nrf2, nuclear erythroid 2-related factor 2; Keap1, Kelch-like ECH-associated protein 1; and MTOR, mammalian target of rapamycin. The values are the means \pm SEM $(n=6){ }^{a, b, c}$ The different superscript letters above the bars show significant differences among the treatments $(P<0.05)$.

$\mathrm{PF}, \mathrm{SF}$, and WF supplementations remarkably increased the SOD1, CAT, $\mathrm{GP}_{X} 1, \mathrm{GR}$, Nrf2, Keap-1, and mTOR mRNA levels in the spleen compared with those in the control diet $(P<0.05)$. Rats supplemented with WF presented a higher SOD1 and CAT mRNA levels compared with those supplemented with PF and SF $(P<0.05)$.

\section{Cytokine mRNA expression profiles in the jejunum, liver, and spleen}

The cytokine mRNA expression profiles of the jejunum are shown in Table 7. PF significantly upregulated IL-1 $\beta$, IL-10, TNF- $\alpha$, and caspase 3 mRNA levels, but downregulated IL-6 mRNA level in the jejunum compared with those of the control group $(P<0.05)$. Rats supplemented with $\mathrm{PF}$ diet exhibited a higher IL-10, TNF- $\alpha$, TGF- $\beta 1$, and caspase 3 mRNA levels compared with those supplemented with SF diet $(P<$ $0.05)$. The IL- $1 \beta$ and caspase 3 mRNA levels in the SF group were significantly higher, but the IL-10 mRNA levels were significantly lower than those in the control and WF groups $(P$ $<0.05)$. The WF group exhibited a significantly higher IL-10 and TGF- $\beta 1$ mRNA expression, but a significantly lower IL-6 and TNF- $\alpha$ mRNA expression than the control group $(P<$ 0.05). Compared with PF diet, WF supplementation resulted in a higher IL-10 mRNA level, but a lower IL-1 $\beta$, TNF- $\alpha$, and caspase 3 mRNA levels in the jejunum $(P<0.05)$.

In liver (Table 8), the IL-1 $\beta$ mRNA levels in the PF and SF groups were significantly downregulated, but the TNF- $\alpha$ mRNA level was upregulated $(P<0.05)$. WF supplementation significantly increased the levels of IL-10 and TGF- $\beta 1$ mRNA expression, but decreased the levels of IL-1 $\beta$ and caspase 3 mRNA expression compared with those of the control group $(P<0.05)$. Higher IL-10 and TGF- $\beta 1$ mRNA levels, but lower TNF- $\alpha$ mRNA level were observed in rats under WF diet compared with those under other diets $(P<0.05)$. 
Table 7 Effects of pea fiber, sweet potato fiber, and wheat bran fiber on the cytokine mRNA expressions of jejunum in rats ${ }^{a, b}$

\begin{tabular}{lllll}
\hline & \multicolumn{2}{l}{ Treatments } & & \\
\cline { 2 - 5 } Parameters & $\mathrm{C}$ & PF & SF & WF \\
\hline IL-1 $\beta$ & $1.00 \pm 0.05^{\mathrm{a}}$ & $2.25 \pm 0.10^{\mathrm{b}}$ & $2.06 \pm 0.07^{\mathrm{b}}$ & $1.10 \pm 0.07^{\mathrm{a}}$ \\
IL-6 & $1.00 \pm 0.12^{\mathrm{b}}$ & $0.40 \pm 0.03^{\mathrm{a}}$ & $0.57 \pm 0.09^{\mathrm{a}}$ & $0.37 \pm 0.01^{\mathrm{a}}$ \\
IL-10 & $1.00 \pm 0.08^{\mathrm{b}}$ & $1.23 \pm 0.07^{\mathrm{c}}$ & $0.72 \pm 0.06^{\mathrm{a}}$ & 0.000 \\
TNF- $\alpha$ & $1.00 \pm 0.06^{\mathrm{b}}$ & $1.33 \pm 0.07^{\mathrm{c}}$ & $0.82 \pm 0.08^{\mathrm{ab}}$ & $0.09^{\mathrm{d}}$ \\
TGF- $\beta 1$ & $1.00 \pm 0.05^{\mathrm{ab}}$ & $1.16 \pm 0.10^{\mathrm{bc}}$ & $0.90 \pm 0.04^{\mathrm{a}}$ & $1.37 \pm 0.04^{\mathrm{a}}$ \\
Casepase 3 & $1.00 \pm 0.04^{\mathrm{a}}$ & $1.71 \pm 0.03^{\mathrm{c}}$ & $1.21 \pm 0.05^{\mathrm{b}}$ & 0.000 \\
\end{tabular}

${ }^{a}$ Data are expressed as mean \pm SEM for eleven rats per treatment group. C, control; PF, pea fiber; SF, sweet potato fiber; WF, wheat bran fiber; IL-1 $\beta$, interleukin $1 \beta$; IL-6, interleukin 6; IL-10, interleukin 10; TNF- $\alpha$, tumor necrosis factor $\alpha$; TGF- $\beta 1$, transforming growth factor $\beta 1 .{ }^{b}$ a-d Mean values within a row with different superscript letters were significantly different $(P<0.05)$.

Table 8 Effects of pea fiber, sweet potato fiber, and wheat bran fiber on the cytokine mRNA expressions of liver in rats ${ }^{a, b}$



${ }^{a}$ Data are expressed as mean \pm SEM for eleven rats per treatment group. C, control; PF, pea fiber; SF, sweet potato fiber; WF, wheat bran fiber; IL-1 $\beta$, interleukin $1 \beta$; IL-10, interleukin 10 ; TNF- $\alpha$, tumor necrosis factor $\alpha$; TGF- $\beta 1$, transforming growth factor $\beta 1 .{ }^{b}{ }^{-c}$ Mean values within a row with different superscript letters were significantly different $(P<0.05)$.

Table 9 Effects of pea fiber, sweet potato fiber, and wheat bran fiber on the cytokine mRNA expressions of spleen in rat ${ }^{a, b}$

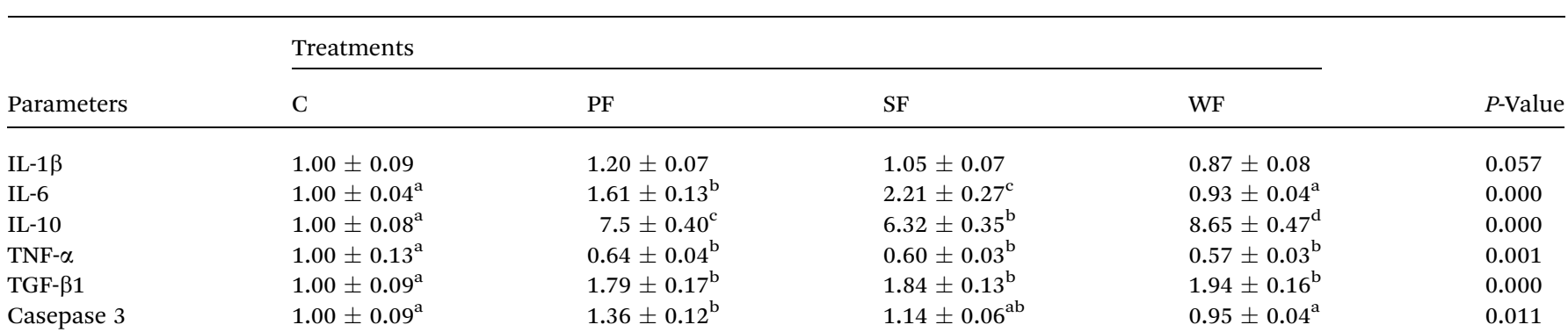

${ }^{a}$ Data are expressed as mean \pm SEM for eleven rats per treatment group. C, control; PF, pea fiber; SF, sweet potato fiber; WF, wheat bran fiber; IL-1 $\beta$, interleukin 1 $\beta$; IL-6, interleukin 6; IL-10, interleukin 10; TNF- $\alpha$, tumor necrosis factor $\alpha$; TGF- $\beta 1$, transforming growth factor $\beta 1 .{ }^{b}$ a-d Mean values within a row with different superscript letters were significantly different $(P<0.05)$.

The PF and SF groups in the spleen exhibited higher levels of IL- 6 , IL-10, and TGF- $\beta 1$ mRNA, but a lower TNF- $\alpha$ mRNA level than those of the control group $(P<0.05)$, as shown in Table 9. Rats fed with SF showed upregulated IL-6 gene expression, but downregulated IL-10 mRNA level compared with the PF and WF groups $(P<0.05)$. Moreover, rats on WF diet showed higher IL-10 and TGF- $\beta 1$ mRNA levels but lower TNF- $\alpha$ mRNA level compared with those fed with the control diet $(P<0.05)$. Rats fed with WF exhibited higher IL-10 gene expression level, but lower but IL- 6 and caspase 3 mRNA levels than those in the PF group $(P<0.05)$.

\section{Discussion}

In human food supply, consumers are increasingly focused on natural sources of antioxidants because of safety concerns associated with supplemental forms. PF, SF, and WF are types of DF that exhibit potential to alter antioxidant status and immune response in vivo and in vitro. To investigate the effect of PF, SF, and WF supplementation on plasma and other relevant organs with antioxidant capacity in rats, we assayed the T-AOC, free radical-scavenging ability (ASA and AHR), activities of enzymatic (T-SOD and CAT) and non-enzymatic (GSH) antioxidants, and gene expression of antioxidant- 
related signaling molecules in the jejunum, liver, and spleen. In addition, the effects of dietary PF, SF, and WF on genes involved in immune cell cytokines of the jejunum, liver, and spleen were analyzed.

\section{Effects of dietary PF, SF, and WF on the antioxidant status in plasma and organs}

The present results showed that the ASA and AHR activities in the liver were enhanced in response to dietary PF, SF, and WF supplementations. Furthermore, rats supplemented with WF diet exhibited higher ASA and AHR activities in the jejunum and liver than those under the SF diet. ASA and AHR activities are two indexes involved in reflecting the total scavenging capacity against $\mathrm{O}_{2}{ }^{-}$and $\mathrm{OH}^{-}$radicals. ${ }^{26}$ The present results indicated that $\mathrm{PF}, \mathrm{SF}$, and WF supplementations can improve the free radical-scavenging ability. In addition, rats fed with WF diet showed improved superoxide anion radical and hydroxyl radical-scavenging capacities. These findings are in agreement with previous studies, which showed that pea, sweet potato, and wheat bran can enhance the free radical-scavenging activity of 1,1-diphenyl-2-picrylhydrazyl. ${ }^{2,6,27}$

The superoxide anion radical $\left(\mathrm{O}_{2}{ }^{-}\right)$and hydroxyl radical $\left(\mathrm{OH}^{-}\right)$scavenging activities in the plasma, jejunum, liver, and spleen may also be associated with the antioxidant defense system. Similar to the superoxide anion and hydroxyl radical scavenging ability in ASA and AHR, the antioxidant enzyme systems (T-SOD and CAT) also exhibit parallel catalytic activity with oxygen radicals. CAT is an excellent endogenous antioxidant enzyme that can eliminate organic hydroxyl radicals, and SOD can collaboratively transform superoxide anions into $\mathrm{H}_{2} \mathrm{O}^{28,29}$ The present study provided evidence of a significant increase in the T-SOD activity in the jejunum and liver of rats under PF- and SF-supplemented groups. Additionally, the jejunum and spleen showed higher CAT and T-SOD activities in the WF-supplemented group compared with those in the SF diet. These results indicated that the activities of antioxidant enzymes were facilitated by dietary PF, SF, and WF in rats. The regulation of fiber source to antioxidant enzyme activities was also related to fiber components as specific promotion substrates. In addition, the specific increase of antioxidant enzyme activities in organs may associate with the enhancement of ASA and AHR activities. ${ }^{30}$

GSH and T-AOC are crucial in preventing oxidative damage. GSH serves as the first line of antioxidant defense system in numerous detoxification reactions, and T-AOC often reflects the endogenous antioxidative capability. ${ }^{31,32}$ In the present study, dietary WF significantly promoted the GSH content and T-AOC in jejunum. Dietary WF and PF can both enhanced the GSH in the spleen. Given these results, we supposed that dietary PF and WF can accelerate the non-enzymatic antioxidant activity in the spleen. Furthermore, the rats supplemented with the PF and WF diets exhibited higher GSH content in the spleen than those in the SF diet. The beneficial effect of dietary PF and WF on nonenzymatic antioxidant activity in the spleen is mainly ascribed to, first, the antioxidant compounds, such as ferulic acid, lignins, and phytic acid in the grain envelope of $\mathrm{DF} ;{ }^{3}$ second, the spleen is the main secondary immune organ in the body and mostly responsible in forming antibodies, differentiating $\mathrm{B}$ cells, and participating in immune responses. ${ }^{33}$ Nevertheless, the mechanisms of antioxidant induction by PF and WF remain unclear.

Taken together, the current results proved that $\mathrm{PF}, \mathrm{SF}$, and WF can be excellent DF candidates to improve the antioxidant ability of organs. Improvements in enzymatic antioxidant activity in organs may be associated with increased gene expression. Thus, we also determined the influence of dietary $\mathrm{PF}, \mathrm{SF}$, and WF on the mRNA levels of antioxidant enzymes in the jejunum, liver, and spleen of rats.

\section{Effects of PF, SF, and WF supplementation on gene expression of antioxidant enzymes in organs}

Dietary WF apparently upregulated the mRNA expression levels of SOD1, CAT, $\mathrm{GP}_{X} 1$, and GR in the jejunum and spleen of rats. Similarly, either dietary PF significantly increased the mRNA levels of SOD1, CAT, and $\mathrm{GP}_{X} 1$, or SF significantly enhanced the SOD1, $\mathrm{GP}_{X}$, and GR mRNA levels in the jejunum and spleen. These results were similar to the increased activity of enzymes. Therefore, the upregulated expression of SOD1, CAT, $\mathrm{GP}_{X} 1$, or GR mRNA of rats following the intake of PF, SF, and WF may be the evidence of increased antioxidant enzyme activities in organs. Moreover, dietary WF provided a higher gene expression of antioxidative enzymes in different tissues compared with those supplemented with other diets. This phenomenon may be partly attributed to the beneficial antioxidant phytochemicals, including phenolic acids, carotenoids, tocopherols, and alkylresorcinols, which are found at the highest concentrations in wheat bran. ${ }^{8}$ Wheat bran feruloyl oligosaccharides can upregulate the mRNA expression levels of SOD, CAT, and HO-1 in organs; ${ }^{34}$ this finding is in agreement with the obtained result for WF in the present study. The upregulation might be caused by the upregulation of the expression of antioxidant enzyme-related cellular signaling molecules in organs. Our study also explored the effects of PF, SF, and WF supplementations on the gene expression of these related signaling molecules in the jejunum, liver, and spleen.

The present results showed that the Nrf2 mRNA level in the liver and spleen was higher in WF group than that in the control group. Nrf2 mRNA levels were the lowest in rats fed with SF diet than in those under PF and WF diets in the jejunum. This result implicated that WF can increase the hepatic and lienic antioxidant enzyme gene expression by promoting the stabilization and activation of Nrf2 in the nucleus. By contrast, the increased antioxidant enzyme level in jejunum may be responsible for Nrf2 degradation by supplementation of PF or SF. Nrf2 modulates the gene expression of several phase II antioxidant enzyme genes and is negatively regulated by the actin-binding protein Keap- $1{ }^{35}$ Consequently, these contrasting changes of Nrf2 expression level in different organs may be related to the changes in Keap-1 mRNA level. PF and SF significantly decreased the mRNA level of Keap-1 in the liver, and PF, SF, and WF significantly enhanced Keap-1 expression level in the jejunum. The Nrf2-Keap1 system is one of the most important 
cellular defensive signaling pathways in animals. ${ }^{11}$ The response can be explained by the Nrf2 expression may be regulated through its upstream signaling molecule of TOR. ${ }^{13}$ However, to our knowledge, no studies have investigated the effect of $\mathrm{PF}, \mathrm{SF}$, and WF on TOR signaling pathway in rats.

To evaluate a potential translational mechanism caused by PF, SF and WF, further experiments were performed to determine the PF-, SF-, and WF-stimulated mTOR level in the jejunum, liver, and spleen. Our data revealed that the mTOR mRNA level in the liver and spleen was significantly increased by PF and WF. MTOR is a cellular signal that participates in cell growth, energy status, and growth factor stimulation. ${ }^{36}$ The upregulation of mTOR expression in the liver and spleen suggested that mTORmediated signaling pathways were activated by PF and WF. The transcription of mTOR genes may respond to the activation of transcription factors such as p53 and FoxO that function downstream of oxidative stress. ${ }^{36}$ The antioxidant function of these fibers seem to be ascribed to their capacity to induce Keap1 degradation and accordingly activate Nrf2 signaling as well as to their ability to accelerate mTOR activity. However, the underlying mechanism in the relationship between different fiber sources and the Nrf2 and mTOR pathways remains largely unknown and requires further investigation.

Overall, the current study demonstrated that a novel mechanism for PF, and WF supplementation can modulate the antioxidant defense through the signaling factors of Nrf2, Keap1 , and mTOR in liver, and spleen. Intake of PF, SF, and WF modulates the antioxidant status in the jejunum, liver, and spleen and may affect local immunity of different organs in rats.

\section{Effects of PF, SF, and WF supplementations on gene expression of inflammatory cytokine in organs}

In this study, we observed that the mRNA levels of hepatic IL-1 $\beta$, jejunal IL-6, and splenic TNF- $\alpha$ in the PF, SF, and WF groups were significantly downregulated. However, the IL-10 mRNA level was upregulated in spleen tissues. WF group exhibited a higher level of IL-10 mRNA, but a lower TNF- $\alpha$ mRNA level in the jejunum and spleen compared with those fed with other diets. These results are consistent with a previous finding that the mRNA expression rates of inflammatory are specific to fiber source and tissue. ${ }^{16}$ Data from studies on pigs also indicated that supplemental DF induces a cellular immune response through regulating cytokine immunomodulation. ${ }^{5}$ IL-1 $\beta$, IL-6, and TNF- $\alpha$ are the typical proinflammatory markers secreted by Th1 cells, and excess amount of these cytokines can damage the host by modifying the production of other cytokines, chemokines, and adhesion molecules. ${ }^{37}$ The anti-inflammatory cytokine IL-10, which is secreted by Th2 cells, is an important regulator of inflammatory responses and critical in T-cell immune response. ${ }^{38}$ Mutual cross inhibition between Th1 and Th2 cytokines polarizes functional Th cell responses into cell-mediated or humoral immune responses. ${ }^{39}$ These data implied that dietary PF, SF, and WF supplementations can enhance rat T-cell immunity function through regulating the mRNA expression of Th1 and Th2 cytokines in organs. WF may also be more beneficial in regulating inflammatory response than PF and SF.
T-cells comprise a group of different T-cell subsets, and TGF$\beta$-expressing Th3 cells are one of the three main subsets of inducible Treg cells. ${ }^{40}$ TGF- $\beta$ is crucial in cell growth, proliferation, differentiation, and apoptosis; this cytokine is also a multifunctional set of peptides that exhibits distinct and essential role in regulating the immune system. ${ }^{41}$ In the present study, WF-supplemented rats exhibited a significantly increased TGF- $\beta 1$ mRNA expression in the jejunum, liver, and spleen. Rats supplemented with WF diet showed a higher TGF- $\beta 1$ mRNA level in the liver compared with those supplemented with SF diet. This result suggested that WF is crucial in the maintenance of healthy immune response in different organs. The remarkably increased expression of TGF- $\beta 1$ in organs of WF diet-fed rats may be the evidence of increased IL-10 mRNA level in those organs. Both TGF- $\beta 1$ and IL-10, which are involved in kill antigen-presenting cells in type 1 Treg cells, suppress the effector T-cell responses. ${ }^{40}$ Furthermore, our data revealed that $\mathrm{PF}$ and SF supplementations significantly increased the caspase 3 mRNA level. Caspase 3 mRNA level in the PF group was also significantly higher than those of the SF and WF groups in the jejunum. Caspase 3 belongs to a family of cysteine proteases called caspases and plays a crucial role in the immune system homeostasis by controlling the programmed cell death process. ${ }^{17}$ The increasing expression of caspase 3 by feeding DF may induce jejunal cell apoptosis; these results disagree with that of a previous study that caspase 3 is downregulated in the colon of pigs receiving PF. ${ }^{42}$ This phenomenon may be related to the effects of PF on caspase 3 gene expression in response to different intestinal segments, and the mechanisms might depend on NF- $\kappa$ B signaling pathway. ${ }^{16}$ These findings also need further investigation.

Taken together, our results highlighted the potential of PF, SF, and WF as excellent candidates to influence immunomodulatory effects and improve immune homeostasis by avoiding excessive immune responses to pathogens and inducing immune tolerance. However, the molecular mechanism has been poorly documented and is completely absent in most cases.

\section{Conclusion}

The present study demonstrated that dietary PF, SF, and WF can effectively promote antioxidant defense by enhancing free radical-scavenging ability, and improving non-enzymatic and enzymatic antioxidant capacities in rats. Moreover, the mRNA expression levels of phase II antioxidant enzymes were regulated by signaling molecules, such as Nrf2, Keap-1, and mTOR in liver and spleen. These results implied that modulation of mTOR pathway may play a significant role in the antioxidant activities of PF, SF, and WF. Furthermore, treatment with PF, $\mathrm{SF}$, and WF can improve immune response through mediating the expression levels of anti-inflammatory and proinflammatory cytokines, TGF- $\beta 1$, and caspase 3 gene. The present results also showed that rats fed with different fiber sources exhibited different qualitative effects on organic antioxidant and immunity functions. WF intake may also reveal a beneficial effect on the normal functions of physiology and 
biochemistry in mammals. This novel and important finding provides a necessary basis for future studies to define the molecular mechanisms and thereby improve the antioxidant capacity and overall immunity function through PF, SF, and WF intake in rats. Finally, this work may provide an important practical implication in determining $\mathrm{PF}, \mathrm{SF}$, and WF as a source of disease-resistant dietary agents.

\section{Acknowledgements}

The authors wish to thank their teammates for kind assistance during experiments. The authors also would like to thank Specific Research Supporting Program for Discipline Construction in Sichuan Agricultural University (to G. Liu) for financial support.

\section{References}

1 A. Papathanasopoulos and M. Camilleri, Gastroenterology, 2010, 138, 65-72.

2 K. X. Zhu, S. Huang, W. Peng, H. F. Qian and H. M. Zhou, Food Res. Int., 2010, 43, 943-948.

3 G. Liu, X. Liang, T. Fang, Y. Cai, G. Jia, H. Zhao, J. Wang, X. Chen and C. Wu, PLoS One, 2014, 9, e115561.

4 G. Liu, G. Yang, T. Fang, Y. Cai, C. Wu, J. Wang, Z. Huang and X. Chen, RSC Adv., 2014, 4, 23749-23758.

5 H. Chen, X. Mao, J. He, B. Yu, Z. Huang, J. Yu, P. Zheng and D. Chen, Br. J. Nutr., 2013, 110, 1837-1848.

6 D. Montserrat, H. Teresa and E. Isabel, Eur. Food Res. Technol., 2007, 225, 493-500.

7 T. Madhujith and S. Fereidoon, J. Food Sci., 2006, 70, S85S90.

8 L. Stevenson, F. Phillips, K. O'Sullivan and J. Walton, Int. J. Food Sci. Nutr., 2012, 63, 1001-1013.

9 M. Kano, T. Takayanagi, K. Harada, K. Makino and F. Ishikawa, Biosci., Biotechnol., Biochem., 2005, 69, 979-988. 10 A. Uruno and H. Motohashi, Nitric Oxide, 2011, 25, 153-160. $11 \mathrm{H}$. Motohashi and M. Yamamoto, Trends Mol. Med., 2004, 10, 549-557.

12 Y. P. Hwang, J. H. Choi, H. J. Yun, E. H. Han, H. G. Kim, Y. K. Jin, B. H. Park, T. Khanal, J. M. Choi and Y. C. Chung, Food Chem. Toxicol., 2010, 49, 93-99.

13 K. P. Shay, A. J. Michels, W. Li, A. N. T. Kong and T. M. Hagen, Biochim. Biophys. Acta, 2012, 1823, 1102-1109.

14 I. J. Elenkov and G. P. Chrousos, Trends Endocrinol. Metab., 1999, 10, 359-368.

15 J. E. Lambert, J. A. Parnell, J. M. Tunnicliffe, J. Han, T. Sturzenegger and R. A. Reimer, Clin. Nutr., 2017, 36, 126-133.

16 S. Kobayashi, Arch. Anim. Nutr., 2008, 62, 427-438.
17 J. Xu, S. Jiang, Y. Li, M. Li, Q. Cheng, D. Zhao, B. Yang, Z. Jia, L. Wang and L. Song, Dev. Comp. Immunol., 2016, 61, 1-12.

18 P. G. Reeves, F. H. Nielsen and F. G. Fahey Jr, J. Nutr., 1993, 123, 1939-1951.

19 T. Fang, G. Liu, W. Cao, X. Wu, G. Jia, H. Zhao, X. Chen, C. Wu and J. Wang, RSC Adv., 2016, 6, 31323-31335.

20 W. D. Jiang, L. Feng, Y. Liu, J. Jiang, K. Hu, S. H. Li and X. Q. Zhou, Food Chem., 2010, 120, 692-697.

21 N. Vardi, H. Parlakpinar, F. Ozturk, B. Ates, M. Gul, A. Cetin, A. Erdogan and A. Otlu, Food Chem. Toxicol., 2008, 46, 30153022 .

22 J. Wan, K. Yang, Q. Xu, D. Chen, B. Yu, Y. Luo and J. He, RSC Adv., 2016, 74, 70715-70722.

23 W. Zhang, Q. Chen, K. Mai, W. Xu, X. Wang and Z. Liufu, Aquacult. Res., 2010, 41, e781-e787.

24 H. Aebi, Methods Enzymol., 1984, 105, 121-126.

25 K. J. Livak and T. D. Schmittgen, Methods, 2001, 25, 402-408. 26 R. Kohen and A. Nyska, Toxicol. Pathol., 2002, 30, 620-650.

27 J. G. Zhao, Q. Q. Yan, R. Y. Xue, J. Zhang and Y. Q. Zhang, Food Chem., 2014, 161, 22-26.

28 M. David, V. Munaswamy, R. Halappa and S. R. Marigoudar, Pestic. Biochem. Physiol., 2008, 92, 15-18.

29 G. W. Winston and R. T. Di Giulio, Aquat. Toxicol., 1991, 19, 137-161.

30 W. Cao, G. Liu, T. Fang, X. Wu, G. Jia, H. Zhao, X. Chen, C. Wu, J. Wang and J. Cai, RSC Adv., 2015, 5, 76607-76614.

31 C. C. Yang, J. Y. Fang, T. L. Hong, T. C. Wang, Y. E. Zhou and T. C. Lin, Int. Immunopharmacol., 2013, 15, 106-113.

32 W. Ren, Y. Yin, G. Liu, X. Yu, Y. Li, G. Yang, T. Li and G. Wu, Amino Acids, 2012, 42, 2089-2094.

33 J. Liu, N. Li, L. Ma, Y. Duan, J. Wang, X. Zhao, S. Wang, H. Wang and F. Hong, J. Alloys Compd., 2010, 489, 708-713.

34 H. Zhang, J. Wang, Y. Liu and B. Sun, Int. J. Biol. Macromol., 2015, 74, 150-154.

35 M. Kobayashi and M. Yamamoto, Antioxid. Redox Signaling, 2005, 7, 385-394.

36 S. G. Rhee and S. H. Bae, Free Radical Biol. Med., 2015, 88, 205-211.

37 V. Wieser, A. R. Moschen and H. Tilg, Arch. Immunol. Ther. Exp., 2013, 61, 119-125.

38 M. A. Engel and M. F. Neurath, Rom. J. Intern. Med., 2015, 53, 95-103.

39 V. A. Depalo, Chest, 2000, 117, 1162-1172.

40 O. Palomares, M. Martínfontecha, R. Lauener, C. Traidlhoffmann, O. Cavkaytar, M. Akdis and C. A. Akdis, Genes Immun., 2014, 15, 511-520.

41 J. J. Letterio and A. B. Roberts, Annu. Rev. Immunol., 1998, 16, 137-161.

42 L. Che, H. Chen, B. Yu, J. He, P. Zheng, X. Mao, J. Yu, Z. Huang and D. Chen, Nutr. Cancer, 2014, 66, 388-399. 